\title{
Partial selfing, ecological disturbance and reproductive assurance in an invasive freshwater snail
}

\author{
P-Y Henry ${ }^{1,3}$, L Bousset ${ }^{1,2}$, P Sourrouille ${ }^{1}$ and P Jarne ${ }^{1}$ \\ ${ }^{1}$ Centre d'Ecologie Fonctionnelle et Evolutive, CNRS, 1919 route de Mende, F-34293 Montpellier cedex 5, France; ${ }^{2} I N R A$, UMR BiO3P, \\ BP 35327, F-35653 Le Rheu cedex, France
}

\begin{abstract}
Although reproductive assurance (RA) might play a central role in the evolution of the selfing rate, this hypothesis has never been seriously investigated in an hermaphroditic animal. We studied the mating system of the freshwater snail Physa acuta in which the availability of mating partners might be highly variable, because this species is an efficient colonizer occupying unstable habitats. A total of 11 populations differing in ecological disturbance regime (water level, openness) and snail densities were monitored over 2 years. The outcrossing rate was estimated in ca 10 families per population using microsatellite markers and the progenyarray approach. Components of fecundity and survival were recorded for each progeny. Predominant outcrossing $\left(t_{\mathrm{m}}=0.94\right)$ was detected, with a few individuals $(4 \%)$ purely
\end{abstract}

selfing. The outcrossing rate did not explain among-family variation in fitness components. None of the predictions formulated under the RA hypothesis were verified: (i) selfing was related neither to disturbed habitats, nor to temporal density fluctuations, (ii) it was positively related to population density, (iii) it co-occurred with multiple paternity, and (iv) it did not induce delayed reproduction. Explanations for these negative results are discussed in light of other arguments supporting the RA hypothesis in $P$. acuta, as well as alternative theories explaining the occurrence of partial selfing, as either a genetically fixed or plastic trait.

Heredity (2005) 95, 428-436. doi:10.1038/sj.hdy.6800731; published online 21 September 2005

Keywords: demography; density; mating system; Physa acuta; reproductive assurance; self-fertilization

\section{Introduction}

The capacity to self-fertilize is obviously advantageous: offspring can be produced even when mates are unavailable. Reproductive assurance (RA) has therefore been a popular hypothesis for explaining the evolution of the selfing rate (see Jarne and Charlesworth, 1993). Further investigations (eg Lande and Schemske, 1985) rationalized the understanding of the genetical processes driving the evolution of the selfing rate, converting the problem mainly into a trade-off between the automatic advantage $(50 \%)$ of gene transmission under selfing and the lowered fitness of selfed relative to outcrossed progeny, that is, inbreeding depression. The influence of environmental constraints has since then been incorporated into several theoretical models that account for population structure and dynamics (eg Ronfort and Couvet, 1995; Pannell and Barrett, 2001; Cheptou and Dieckmann, 2002). Theoretical studies focusing specifically on the role of the reproductive assurance hypothesis (RAH) showed that delayed selfing is always advantageous when it occurs as RA (Lloyd, 1992; Tsitrone et al, 2003a), even in a metapopulational context (Pannell and Barrett, 1998; but see Pannell and Barrett, 2001).

Correspondence: P-Y Henry, Centre d'Ecologie Fonctionnelle et Evolutive, CNRS, 1919 route de Mende, F-34293 Montpellier cedex 5, France.

E-mail: philippe.jarne@cefe.cnrs.fr

${ }^{3}$ Current address: Department of Ecology and Evolutionary Biology, 301 Guyot Hall, Princeton University, Princeton, NJ 08544, USA.

Received 6 October 2004; accepted 15 July 2005; published online 21 September 2005
The wide acceptance of the central role of RA in the evolution of selfing (eg Lloyd, 1980; Schoen et al, 1996; Barrett, 2002) basically relies on empirical evidence. Major arguments are the over-representation of selfcompatible species among long-distance colonizing species (reviewed in Pannell and Barrett, 1998), the repeated evolution of selfing populations or species from outcrossing ancestors in constraining habitats (eg Dole, 1992), and the occurrence of autogamous characters in populations experiencing chronically low density (eg Dole, 1992; Städler and Jarne, 1997). More direct arguments are derived from studies on plant pollination showing that selfing correlates with pollen-vector unreliability and/or low plant population density (eg Lloyd, 1980; Watkins and Levin, 1990; Fausto et al, 2001). However, the evolutionary significance of the RAH has been recently questioned (eg Barrett, 2002), the advantage of selfing being outweighed by seed discounting and inbreeding depression (Herlihy and Eckert, 2002). Moreover, although widely studied in plants, RA has hardly been investigated in hermaphroditic animals.

We engaged in an ecological and genetical study aiming at evaluating the role of RA in a hermaphroditic freshwater snail, Physa acuta (Gastropoda: Pulmonata; Henry, 2002; Tsitrone et al, 2003b; Bousset et al, 2004). Freshwater snails are good candidates for such a work (Jarne and Charlesworth, 1993; Städler and Jarne, 1997). $P$. acuta predominantly outcrosses, probably because of strong inbreeding depression (Jarne et al, 2000; Henry et al, 2003; Tsitrone et al, 2003b). Residual selfing in outcrossing animal species might constitute an adaptive 
strategy of RA (Tsitrone et al, 2003a), and there are some ecological reasons for thinking that it might be so in $P$. acuta. First, this species inhabits a wide range of more or less temporary habitats varying in openness (eg ponds versus rivers). As a consequence, population size is likely to vary dramatically through time because of drying out events or floods (reviewed in Städler and Jarne, 1997). Second, $P$. acuta is the commonest species in isolated habitats (ie ponds) of the area studied here (reviewed in Henry, 2002), in accordance with its invasive status (Dillon et al, 2002; Bousset and Jarne, unpublished data). As colonization has been shown to be associated with founding events in freshwater snails (Charbonnel et al, 2002; Meunier et al, 2004), this opens opportunities for selfing as a RA strategy. In this context, Tsitrone et al (2003a) have shown theoretically that a key-factor, if RA is to play a role in the evolution of selfing, is the waiting time - the time that an isolated individual should wait before selfing when compared to an outcrossing individual. Its occurrence has been experimentally documented, and this character proved to be heritable (Tsitrone et al, 2003b; Escobar, David and Jarne, unpublished data). This direct approach suggests that the RAH can explain residual selfing rates in outcrossing species.

The RAH is here tested through an indirect, correlative approach, aiming at determining whether population and individual variation in selfing rate are affected by environmental, demographic and individual factors as predicted under the RAH. In all, 11 sites harbouring populations of $P$. acuta were studied representing four habitat types characterized by their water regime (temporary versus permanent) and openness (ponds versus rivers). Population density and water level were monitored monthly for 2 years. The outcrossing rate was estimated in $\mathrm{ca} 10$ families per population using microsatellite markers and the progeny-array approach (Ritland, 2002).

Predictions from the RAH were formulated under two models for the genetic basis of selfing. First, selfing was assumed to be a genetically fixed trait, with natural selection acting on the selfing rate. The selfing rate would be adapted to the past history of mate availability. The RAH predicted that mate availability and the outcrossing rate covary across ecological disturbance regimes (water regime and openness), both being lower in temporary than in permanent habitats, and in ponds than in rivers. Second, selfing was assumed to be a genetically plastic trait, with natural selection acting on the adjustment of the selfing rate to environmental conditions (which is possibly the case in P. acuta; Tsitrone et al, 2003b). The selfing rate would thus be adapted to the instantaneous mate availability. Predictions from the RAH were: the outcrossing rate increases with increasing density, multiple paternity is positively associated with the outcrossing rate, and the time to first egg laying of isolated individuals under laboratory conditions is negatively associated with the outcrossing rate.

\section{Materials and methods}

Populations studied and progeny arrays

A total of 11 populations of $P$. acuta were monitored monthly from July 2000 to 2002 in the Montpellier area, France. Ecological details of these populations are given in Table 1 (see also Henry, 2002). Analysis of population genetic structure is presented in Bousset et al (2004). Populations were distinguished according to habitat openness (rivers = open versus ponds=closed) and water regime (permanent versus temporary). Temporary sites dried out at least once during the 2-year survey. The snail density was visually scored ( $c a 0,1,5,10,25,50$ or $100 \mathrm{ind} / \mathrm{m}^{2}$ ) at each visit. When snails were spatially aggregated, we reported the range of density $\left(d_{\text {sampl }}\right.$ in Table 1), and the range mean was used in statistical analyses.

In July 2000, 20 mature individuals (denoted $\mathrm{G}_{0}$ ) were collected in each population. They were measured (length) and isolated in $75 \mathrm{ml}$ plastic boxes less than $6 \mathrm{~h}$ after field collection. They were maintained in the laboratory at $25^{\circ} \mathrm{C}$ under a $12: 12$ photoperiod regime and fed ad libitum with boiled lettuce. Water was changed every 3 days. Plastic boxes were checked daily for egg capsules, until the first capsule was laid, over a period of 21 days. The egg-laying delay ( $t_{\text {laying; mean: }}$ $5.1 \pm 3.9$ days) was calculated as the time elapsed between isolation and first egg laying. The fraction of fertile individuals $\left(p_{\text {fert }}\right)$ was the fraction of individuals that laid eggs over these 21 days. When several capsules were laid by a given snail, only the oldest capsule, based on embryo development, was analysed. Its number of eggs $\left(N_{\text {egg }}\right)$ was recorded. Hatching was checked daily until the first juvenile (denoted $\mathrm{G}_{1}$ ) was detected, from which we derived the minimum incubation time $\left(t_{\text {incub }}\right)$, that is, the time elapsed between egg laying and first hatching. Survival $\left(S_{7 \mathrm{~d}}\right)$ was defined as the number of surviving $G_{1} 7$ days after first hatching divided by the number of eggs per capsule.

A $G_{0}$ mother and its $G_{1}$ offspring constituted a family. Since P. acuta stores sperm after copulation (eg Wethington and Dillon, 1997), preferentially outcrosses (Jarne et al, 2000; Tsitrone et al, 2003b; Bousset et al, 2004) and $\mathrm{G}_{0}$ individuals were placed in optimal conditions for egg laying, we assumed that the estimated outcrossing rate reflects that under natural conditions.

\section{Estimating the outcrossing rate}

$\mathrm{G}_{0}$ and $\mathrm{G}_{1}$ individuals were stored at $-80^{\circ} \mathrm{C}$ and in $95 \%$ alcohol, respectively, prior to DNA extraction. DNA extraction, PCR amplification and allele size scoring were performed according to Sourrouille et al (2003). The seven loci used are described in Monsutti and Perrin (1999) and Sourrouille et al (2003). Loci were coamplified by PCR (Pac1, Pac2 and Pac5; Pac4 and Pac7; AF108762 and AF108764; Bousset et al, 2004) and products were run simultaneously on ABI Prism Genetic Analysers (310 or 3100). PCR amplification and allele size scoring were performed simultaneously for the $G_{0}$ mother and its progeny. In all, 10 families per population and seven juveniles per family were genotyped. When less than seven juveniles were available, at least one family was added in order to genotype the same overall number of juveniles per population (Table 1). Families and juveniles were randomly chosen. Mendelian segregation of alleles at the loci used was checked elsewhere (Bousset et al, 2004). When null alleles were detected (loci Pac4, Pac7), data from these families and loci were omitted from the analysis. Estimates of allelic frequencies per locus and per population are given in Web Table 1, available online. 
Table 1 Ecological characterization, population and individual covariates, and mating system parameters estimates (with SD in parentheses) for 11 populations of Physa acuta

\begin{tabular}{|c|c|c|c|c|c|c|c|c|c|c|c|}
\hline Population & Sal2 & MosI & Lam1 & Lam12 & Mos6 & Vio2 & Vio7 & Vio11 & Vio1 & Vio8 & Vio12 \\
\hline Openness & $\mathrm{O}$ & $\mathrm{O}$ & $\mathrm{O}$ & $\mathrm{O}$ & $\mathrm{O}$ & $\mathrm{C}$ & $\mathrm{C}$ & $\mathrm{C}$ & C & $\mathrm{C}$ & $\mathrm{C}$ \\
\hline Water regime & $\mathrm{P}$ & $\mathrm{P}$ & $\mathrm{T}$ & $\mathrm{T}$ & $\mathrm{T}$ & $\mathrm{P}$ & $\mathrm{P}$ & $\mathrm{P}$ & $\mathrm{T}$ & $\mathrm{T}$ & $\mathrm{T}$ \\
\hline$d_{\text {sampl }}$ & $10-25$ & 25 & $5-100$ & 100 & 1 & 1 & 100 & 25 & 50-100 & 10 & 25 \\
\hline$d_{\text {harm }}$ & 4.73 & 0.66 & 0.36 & 0.23 & 0.45 & 0.61 & 0.95 & 0.54 & 11.22 & 15.45 & 0.84 \\
\hline$d_{\text {freq }}$ & 0.12 & 0.00 & 0.56 & 0.00 & 0.36 & 0.52 & 0.28 & 0.26 & 0.04 & 0.00 & 0.24 \\
\hline$p_{\text {fert }}$ & 0.900 & 0.789 & 0.941 & 0.850 & 0.833 & 0.526 & 0.842 & 0.500 & 0.833 & 0.875 & 0.895 \\
\hline Size & $7.6(0.8)$ & $8.0(1.1)$ & $8.5(0.5)$ & $8.9(0.7)$ & $10.8(3.8)$ & $8.5(1.0)$ & $8.6(0.6)$ & $8.6(1.0)$ & $8.1(0.9)$ & $9.7(0.8)$ & $10.7(3.0)$ \\
\hline$N_{\mathrm{egg}}$ & $12.6(5.3)$ & $18.5(8.8)$ & $25.0(11.9)$ & $12.5(6.23)$ & 36.7 (14.6) & $22.6(13.1)$ & $25.2(8.8)$ & $29.8(4.1)$ & $15.0(5.5)$ & $25.0(9.1)$ & $28.9(9.8)$ \\
\hline$t_{\text {laying }}$ & $2.6(1.9)$ & 8.9 (3.5) & $5.6(3.8)$ & $6.7(4.8)$ & $2.3(0.6)$ & $4.4(5.3)$ & $6.5(5.8)$ & $5.5(2.3)$ & $4.6(4.4)$ & $8.3(3.6)$ & $3.0(1.1)$ \\
\hline$t_{\text {incub }}$ & $5.8(0.8)$ & $6.5(1.1)$ & $8.1(1.1)$ & $5.8(0.7)$ & $5.3(0.6)$ & $6.0(1.0)$ & $8.4(2.7)$ & $6.4(0.9)$ & $5.7(0.6)$ & $6.4(1.1)$ & $8.1(2.9)$ \\
\hline$S_{7 \mathrm{~d}}$ & $0.739(0.250)$ & $0.814(0.219)$ & $0.821(0.143)$ & $0.819(0.282)$ & $0.878(0.144)$ & $0.832(0.232)$ & $0.635(0.311)$ & $0.676(0.277)$ & $0.884(0.142)$ & $0.760(0.211)$ & $0.537(0.295)$ \\
\hline$N_{\text {fam }}$ & 13 & 11 & 10 & 12 & 3 & 7 & 11 & 6 & 11 & 10 & 12 \\
\hline$N_{\mathrm{G}_{0}}$ & 19 & 18 & 16 & 17 & 4 & 18 & 19 & 18 & 17 & 14 & 17 \\
\hline$N_{\mathrm{G}_{1}}$ & 80 & 76 & 70 & 75 & 21 & 44 & 71 & 43 & 76 & 70 & 79 \\
\hline$F_{\text {is }}$ & $0.040(0.047)$ & $0.047(0.044)$ & $0.006(0.020)$ & $0.007(0.022)$ & $0.047(0.066)$ & $0.210(0.076)^{*}$ & $0.088(0.069)^{*}$ & $0.001(0.005)$ & $0.098(0.065)^{*}$ & $0.115(0.051)^{*}$ & $0.011(0.022)$ \\
\hline$t_{F_{\mathrm{sis}}}$ & 0.923 & 0.910 & 0.988 & 0.986 & 0.910 & 0.653 & 0.838 & 0.998 & 0.821 & 0.794 & 0.978 \\
\hline$t_{\mathrm{m}}$ & $0.991(0.011)$ & $0.977(0.026)$ & $0.990(0.013)$ & $0.834(0.100)^{*}$ & $0.998(0.045)$ & $0.957(0.060)$ & $0.731(0.135)^{*}$ & $0.974(0.042)$ & $0.847(0.083)^{*}$ & $0.998(0.011)$ & $0.997(0.015)$ \\
\hline$t_{\mathrm{s}}$ & $0.970(0.025)$ & $0.926(0.042)^{*}$ & $0.935(0.043)^{*}$ & $0.876(0.108)^{*}$ & $0.998(0.045)$ & $0.926(0.094)$ & $0.664(0.157)^{*}$ & $0.945(0.048)^{*}$ & $0.726(0.108)^{*}$ & $0.987(0.023)$ & $0.971(0.019)^{*}$ \\
\hline$t_{\mathrm{m}}-t_{\mathrm{s}}$ & $0.021(0.020)$ & $0.051(0.036)$ & $0.055(0.039)^{*}$ & $-0.042(0.057)$ & $0.000(0.000)$ & $0.031(0.050)$ & $0.067(0.039)^{*}$ & $0.029(0.026)^{*}$ & $0.120(0.045)^{*}$ & $0.011(0.023)$ & $0.025(0.015)^{*}$ \\
\hline$r_{\mathrm{s}}$ & $0.063(0.028)^{*}$ & $0.253(0.098)^{*}$ & $0.078(0.038)^{*}$ & $0.845(0.158)^{*}$ & $0.104(0.041)^{*}$ & $0.618(0.273)^{*}$ & $0.652(0.270)^{*}$ & $0.063(0.122)$ & $0.406(0.286)^{*}$ & $0.103(0.010)^{*}$ & $0.104(0.011)^{*}$ \\
\hline$r_{\mathrm{p}}$ & $0.321(0.142)^{*}$ & $0.200(0.117)^{*}$ & $0.279(0.093)^{*}$ & $0.629(0.207)^{*}$ & $0.028(0.052)^{*}$ & $0.445(0.362)$ & $0.290(0.171)^{*}$ & $0.038(0.099)^{*}$ & $0.125(0.062)^{*}$ & $0.175(0.093)^{*}$ & $0.084(0.048)^{*}$ \\
\hline
\end{tabular}

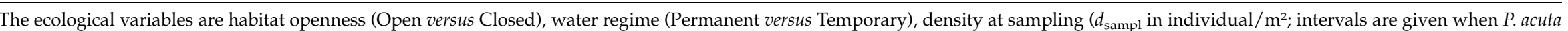
were spatially aggregated), harmonic mean of population densities $\left(d_{\text {harm }}\right)$, and frequency of occurrence of population densities of one ind $/ \mathrm{m}^{2}$ or less $\left(d_{\text {freq }}\right)$. A population covariate is the proportion of fertile individuals ( $\left.p_{\text {fert }}\right)$. The individual covariates are the size of $\mathrm{G}_{0}$ individuals $(\mathrm{mm})$, number of eggs per capsule $\left(N_{\text {egg }}\right)$, egg-laying delay $\left(t_{\text {laying }}\right.$ in days), incubating time $\left(t_{\text {incub }}\right.$ in days), and survival 7 days after first hatching $\left(S_{7 \mathrm{~d}}\right) . N_{\mathrm{fam}}, N_{\mathrm{G}_{0}}$ and $N_{\mathrm{G}_{1}}$ are the number of families, of $\mathrm{G}_{0}$ and $\mathrm{G}_{1}$ individuals genotyped. $F_{\text {is }}$ is the inbreeding coefficient, and $t_{F_{\mathrm{is}}}$ the population outcrossing rates based on $F_{\mathrm{is}} . t_{\mathrm{m}}$ and $t_{\mathrm{s}}$ are the multilocus and single-locus outcrossing rates, and $t_{\mathrm{m}}-t_{\mathrm{s}}$ the amount of apparent selfing accounted for by biparental inbreeding. $r_{\mathrm{s}}$ and $r_{\mathrm{p}}$ are the correlations of selfing among families and of outcrossed paternity within families. Stars indicate estimates significantly higher than $0\left(F_{\mathrm{is},} t_{\mathrm{m}}-t_{\mathrm{s}}, r_{\mathrm{s}}\right)$ or lower than $1\left(t_{\mathrm{m}}, t_{\mathrm{s},} r_{\mathrm{p}}\right)$. 
The mean number of polymorphic loci per population ranged between 3 and 6 , and the mean number of alleles per loci and population varied between 1.8 and 4.2 (Bousset et al, 2004).

The mating system parameters were estimated using maximum likelihood procedures (software MLTR 2.4; Ritland, 2002). Both population-level multilocus $\left(t_{\mathrm{m}}\right)$ and single-locus $\left(t_{\mathrm{s}}\right)$ outcrossing rates were estimated. Family-level multilocus outcrossing rates are noted $t_{\mathrm{mf}}$. The difference between $t_{\mathrm{m}}$ and $t_{\mathrm{s}}$ gives a minimum estimate of apparent selfing due to biparental inbreeding (Ritland, 2002). Correlations of selfing $\left(r_{\mathrm{s}}\right)$ document the among-family variation in selfing, with a non-null correlation indicating significant heterogeneity (Ritland, 2002). Correlations of paternity $\left(r_{\mathrm{p}}\right)$ represent the proportions of full-sibs (versus half-sibs) among outcrossed offspring (Sun and Ritland, 1998; Ritland, 2002) with $r_{\mathrm{p}}$ significantly lower than 1 indicating multiple paternity. The Expectation-Maximization method and the 'parent chosen in probability' option were used. SD of estimates were calculated based on 500 bootstraps assuming Normal distributions. The resampling unit was, respectively, the family and the individual within family for population- and family-level estimates. SD for averages over all population or family estimates were computed with the $\delta$-method (Mood et al, 1974). $F_{\text {is }}, t_{\mathrm{m}}-t_{\mathrm{s}}$ and $r_{\mathrm{s}}$ were considered as significantly higher than 0 , and $t_{\mathrm{m}}, t_{\mathrm{s}}$ and $r_{\mathrm{p}}$ as lower than 1 when more than $95 \%$ of the 500 resampled estimates were, respectively, higher than 0 or lower than 1. Assuming that selfing is the sole source of inbreeding, we calculated the selfing rate corresponding to $F_{\text {is }}$ values according to: $t_{F_{\text {is }}}=\left(1-F_{\text {is }}\right) /\left(1+F_{\text {is }}\right)$ (Pollak, 1987).

\section{Selfing rates and fitness traits}

Since juveniles were genotyped 7 days after hatching, $t_{\mathrm{m}}$ and $t_{\mathrm{mf}}$ estimates are expected to be biased positively, because of inbreeding depression (Jarne and Charlesworth, 1993). This is particularly likely since inbreeding depression is strong in juvenile P. acuta (Wethington and Dillon, 1997; Jarne et al, 2000; Henry et al, 2003; Tsitrone et $a l, 2003 \mathrm{~b})$. The $t_{\mathrm{m}}$ value should therefore explain population-level variation in the proportion of fertile $G_{0}$ individuals $\left(p_{\text {fert }}\right)$, and $t_{\mathrm{mf}}$ should explain family-level variation in the number of eggs per capsule $\left(N_{\mathrm{egg}}\right)$, incubating time $\left(t_{\text {incub }}\right)$ and survival at 7 days $\left(S_{7 \mathrm{~d}}\right)$.

\section{Tests of the RAH}

Predictions from the RAH depend on the assumed genetic basis of the selfing rate (see Introduction; Table 2). (i) The selfing rate could be a genetically fixed trait (predictions prefixed by F) evolving under the selective pressure of temporal fluctuation in mate availability. Mate availability was expected to differ with habitat. Episodes of low density should be less frequent in permanent than in temporary habitats, because population bottlenecks associated with drying-out events are expected to be more frequent in temporary habitats (Charbonnel et al, 2002; Trouvé et al, 2003; Bousset et al, 2004). They should also be more frequent in closed than in open habitats, because immigration more easily refills populations after a population bottleneck in open than in closed habitats (Bousset et al, 2004). The probability of low density episodes was estimated based on the
Table 2 Seven predictions (numbered as in text) relating the outcrossing rate $(t)$ to ecological characteristics, and fitness traits, as derived from the reproductive assurance hypothesis

\begin{tabular}{lllr}
\hline Number & Level & & Prediction \\
\hline F1 & Population & Contrast & $t_{\text {permanent }}>t_{\text {temporary }}$ \\
F2 & Population & Contrast & $t_{\text {open }}>t_{\text {closed }}$ \\
F3 & Population & Correlation & $\left(t, d_{\text {harm }}\right)>0$ \\
F4 & Population & Correlation & $\left(t, d_{\text {freq }}\right)<0$ \\
P1 & Population & Correlation & $\left(t, d_{\text {sampl }}\right)>0$ \\
P2 & Family & Correlation & $\left(t, r_{\mathrm{p}}\right)<0$ \\
P3 & Family & Correlation & $\left(t, t_{\text {laying }}\right)<0$ \\
\hline
\end{tabular}

Predictions were derived under either fixed (noted F) or plastic (noted P) genetic determination of selfing. Habitats were characterized as permanent or temporary, and as open or closed. The outcrossing rate (and therefore the prediction) is either at the population, or at the family level.

$d_{\text {sampl }}$ : density at sampling; $d_{\text {harm }}$ : harmonic mean of population densities; $d_{\text {freq }}$ : frequency of occurrence of population densities of one ind $/ \mathrm{m}^{2}$ or less; $r_{\mathrm{p}}$ : correlation of outcrossed paternity within families; $t_{\text {laying: }}$ time elapsed between isolation and first egg laying.

harmonic mean density $\left(d_{\text {harm }}\right)$ and the proportion of visits when density was one individual per $\mathrm{m}^{2}$ or less $\left(d_{\text {freq }}\right)$ over our 2-year survey. This threshold was chosen because we were technically unable to estimate density precisely at lower values. In response to these expected variations of mate availability, the outcrossing rate $t_{\mathrm{m}}$ was predicted to be higher in permanent than in temporary habitats (F1), in open than in closed habitats (F2), positively correlated to $d_{\text {harm }}$ (F3), and negatively correlated to $d_{\text {freq }}(\mathrm{F} 4)$.

(ii) The selfing rate could be a plastic trait adapted to realise the best compromise between inbreeding depression and instantaneous mate availability (predictions prefixed by P). (P1) At the population level, the probability of outcrossing should be positively correlated with partner availability, estimated as local density during genetic sampling $\left(d_{\text {sampl }}\right)$. (P2) Under delayed selfing, partner availability should not only affect the fraction of selfed ovules, but also the number of outcrossing partners. The probability of outcrossing should thus be negatively correlated to the proportion of half-sibs among outcrossed offspring, $r_{\mathrm{p}}$. (P3) Previous work has shown the existence of a waiting time in $P$. acuta, defined as the difference in age at first egg-laying between outcrossing and selfing virgin individuals (Tsitrone et al, 2003a,b). By analogy, the individual egglaying delay ( $t_{\text {laying }}$ ) was expected to negatively correlate with the outcrossing rate.

\section{Statistical analyses}

The probability of outcrossing was analysed using logistic regression models, followed by likelihood ratio tests (LRT). To convert the normal ML estimates of outcrossing rate $t_{\mathrm{m}}$ into a binomial variable, that is, the probability of being an outcrossed offspring, the number of genotyped progeny per population $N_{\mathrm{G} 1}$ was multiplied by $t_{\mathrm{m}}$ and $1-t_{\mathrm{m}}$ (Table 1 ). This produced the estimated number of outcrossed and selfed juveniles per population. Using logistic regressions, with $t_{\mathrm{m}}$ as the dependent variable and the reconstructed number of juveniles as sample size, accounted for the binomial variance of $t_{\mathrm{m}}$ estimates. Note that this is not the case 
Table 3 Analysis of fitness components and outcrossing rate in P. acuta

\begin{tabular}{|c|c|c|c|c|c|c|c|c|}
\hline \multirow[t]{2}{*}{ Fitness component } & \multicolumn{2}{|c|}{$\operatorname{Ln}\left(\mathrm{t}_{\text {laying }}+1\right)$} & \multicolumn{2}{|c|}{$\operatorname{Ln}\left(\mathrm{N}_{e g g}\right)$} & \multicolumn{2}{|c|}{$\operatorname{Ln}\left(\mathrm{t}_{\text {incub }}\right)$} & \multicolumn{2}{|c|}{$\mathrm{S}_{7 d}$} \\
\hline & $\mathrm{F}(1,96)$ & $\mathrm{P}$ & $\mathrm{F}(1,96)$ & $\mathrm{P}$ & $\mathrm{F}(1,92)$ & $\mathrm{P}$ & $\mathrm{F}(1,91)$ & $\mathrm{P}$ \\
\hline Ln(size) & 0.014 & 0.906 & 0.001 & 0.972 & 0.169 & 0.682 & 0.382 & 0.538 \\
\hline $\operatorname{Ln}\left(N_{\text {egg }}\right)$ & 0.030 & 0.863 & - & - & 0.257 & 0.614 & 2.824 & 0.096 \\
\hline $\operatorname{Ln}\left(t_{\text {laying }}+1\right)$ & - & - & 0.605 & 0.439 & 0.001 & 0.976 & - & - \\
\hline$t_{\mathrm{mf}}$ & 0.460 & 0.499 & 0.169 & 0.682 & 0.383 & 0.537 & 0.556 & 0.458 \\
\hline $\operatorname{Ln}($ size $) * \operatorname{Ln}\left(N_{\text {egg }}\right)$ & 0.205 & 0.651 & - & - & 0.452 & 0.503 & 1.906 & 0.171 \\
\hline $\operatorname{Ln}($ size $) * \operatorname{Ln}\left(t_{\text {laying }}+1\right)$ & - & - & 0.225 & 0.637 & 0.131 & 0.718 & - & - \\
\hline $\operatorname{Ln}($ size $) * t_{\mathrm{mf}}$ & 0.036 & 0.851 & 0.044 & 0.834 & 0.119 & 0.731 & 0.710 & 0.402 \\
\hline $\operatorname{Ln}\left(N_{\text {egg }}\right) * \operatorname{Ln}\left(t_{\text {laying }}+1\right)$ & - & - & - & - & 0.254 & 0.615 & - & - \\
\hline $\operatorname{Ln}\left(N_{\text {egg }}\right) * t_{\mathrm{mf}}$ & 2.104 & 0.150 & - & - & 0.301 & 0.584 & 0.366 & 0.547 \\
\hline $\operatorname{Ln}\left(t_{\text {laying }}+1\right) * t_{\mathrm{mf}}$ & - & - & 1.827 & 0.180 & 0.273 & 0.603 & - & - \\
\hline
\end{tabular}

The table reports the $F$-values and their associated probability $(P)$. The dependent variables (first row) are the log-transformed egg-laying delay ( $t_{\text {laying; }}$ since individuals laid eggs within 1 day, the dependent variable was $\left.\operatorname{Ln}\left(t_{\text {laying }}+1\right)\right)$, number of eggs per capsule $\left(N_{\text {egg }}\right)$, minimum incubating time $\left(t_{\text {incub }}\right)$ and survival at 7 days $\left(S_{7 \mathrm{~d}}\right)$. The first three variables were analysed with ANOVA models and $S_{7 \mathrm{~d}}$ with a logistic regression model. The explanatory variables were the log-transformed individual size, $\operatorname{Ln}\left(N_{\text {egg }}\right), \operatorname{Ln}\left(t_{\text {laying }}+1\right)$ and the family-level multilocus outcrossing rate $t_{\mathrm{mf}}$. Interaction terms are denoted by a star. - indicates that the effect was not included in the model.

under analyses of variance (ANOVA) with classical transformation (eg arcsin) of $t_{\mathrm{m}}$ which, however, provided similar results (results not shown). The explanatory variables were habitat openness, water regime and density variables $\left(d_{\text {harm }}, d_{\text {freq }}\right.$ and $\left.d_{\text {sampl }}\right)$. The effects of openness, water regime and their interaction were tested in a single model, and that of density variables in separate models.

The dependent variables $d_{\text {freq, }}, p_{\text {fert }}$ and $S_{7 \mathrm{~d}}$ were also analysed using logistic regression models: $d_{\text {freq }}$ was modelled in relation to openness, water regime and their interaction, and $p_{\text {fert }}$ in relation to $t_{\mathrm{m}}$. Family-level $\operatorname{Ln}\left(t_{\text {laying }}+1\right), \operatorname{Ln}\left(N_{\text {egg }}\right)$, and $\operatorname{Ln}\left(t_{\text {incub }}\right)$ were analysed using ANOVAs. The explanatory variables for $S_{7 \mathrm{~d}}$, $\operatorname{Ln}\left(t_{\text {laying }}+1\right), \operatorname{Ln}\left(N_{\text {egg }}\right)$ and $\operatorname{Ln}\left(t_{\text {incub }}\right)$ were the $\log$ transformed individual size, $t_{\text {laying }}+1, N_{\text {egg }}, t_{\text {incub }}$ and $t_{\mathrm{mf}}$ (see Table 3 for more details on each model). The homogeneity of $d_{\text {harm }}$ among habitats was tested using a Kruskal-Wallis test, followed by Mann-Whitney tests of the predicted relationships with habitat openness and water regime. Logistic regression and ANOVA models were built with JUMP 3.2.1 (S.A.S. Institute Inc.), and nonparametric tests with Statistica 5.5 (Statsoft Inc.).

\section{Results}

\section{Mating system characterization}

$P$. acuta preferentially outcrossed in all populations. Population-level multilocus outcrossing rates ranged from 0.731 to 0.998 (average: $0.936 \pm 0.019$ ). The selfing rate significantly differed from 0 in three populations out of 11 (Lam12, Vio7, Vio1; Table 1). However, the correlation of selfing $\left(r_{\mathrm{s}}\right)$ among families indicated that outcrossing rates significantly varied among families in 10 populations out of 11 (Table 1). The family-level outcrossing rates ranged from 0.001 to 1 (average: $0.885 \pm 0.002)$. Partial selfing was detected in at least one family per population (except in Mos6), and purely selfed families occurred in three populations only (Lam12, Vio7 and Vio1). $t_{\mathrm{m}}-t_{\mathrm{s}}$ ranged from -0.042 to
0.12 (average: $0.041 \pm 0.011$ ) with significant biparental inbreeding occurring in five populations (significant $t_{\mathrm{m}}-$ $t_{\mathrm{s}}$ in Table 1). $F_{\text {is }}$ differed from zero in four populations out of 11 (range: 0.088-0.210). However, no obvious relationship could be established with inbreeding since $F_{\text {is }}$ was significant in Vio2 and Vio8 where neither selfing, nor biparental inbreeding was detected. Population-level correlations of paternity $\left(r_{\mathrm{p}}\right)$ ranged from 0.028 to 0.629 (Table 1), indicating multiple paternities within progenies in all populations but Vio2. The family-level average was $0.260 \pm 0.613$ (range: $0.001-1$ ) corresponding to a mean number of 3.8 fathers per family (ie $1 / r_{p}$; Sun and Ritland, 1998).

\section{Selfing rates and fitness traits}

The logistic regression model for $S_{7 \mathrm{~d}}$ did not fit the data $\left(\chi^{2}=576.3\right.$, d.f. $\left.=91, P<10^{-4}\right)$. Deviances were thus divided by a variance inflation factor $\hat{c}$ (deviance/ d.f. $=6.333$ ) before testing effects with LRTs. No effect of the outcrossing rate on any of the four reproductive parameters studied was detected among families (Table 3). This also holds for the proportion of fertile individuals (LRT $=0.189$, d.f. $=1, P=0.664$ ). None of the individual traits expected to confound the effect of the mating system significantly explained variation in fitness components (Table 3 ).

\section{Tests of the RAH}

Mate availability depended on the disturbance regime. The harmonic mean density $\left(d_{\text {harm }}\right)$ differed among the four habitats $(H=7.833$, d.f. $=3, N=11, P=0.050)$ with slightly higher values in closed temporary than in other sites (Table 1). When habitat types were tested separately, there was no detectable effect for either habitat openness $(U=6, Z=-1.643, P=0.100)$, or for water regime $(U=13, Z=0.365, P=0.715)$. The probability of occurrence of densities of one individual or less per $\mathrm{m}^{2}\left(d_{\text {freq }}\right)$ varied among habitats (interaction Openness*Water regime, $\mathrm{LRT}=29.818$, d.f. $\left.=1, P<10^{-4}\right)$. However, it did not follow the predicted pattern: low densities were 
Table 4 Likelihood-ratio tests (LRT) for ecological correlates of the population-level outcrossing probability

\begin{tabular}{lrcc}
\hline & $L R T$ & $d . f$. & $\mathrm{P}$ \\
\hline Openness & 5.958 & 1 & 0.015 \\
Water regime & 0.313 & 1 & 0.576 \\
Openness*water regime & 13.849 & 1 & 0.001 \\
$d_{\text {harm }}$ & 0.364 & 1 & 0.546 \\
$d_{\text {freq }}$ & 1.570 & 1 & 0.210 \\
$d_{\text {sampl }}$ & 64.680 & 1 & $<10^{-4}$ \\
\hline
\end{tabular}

The dependent variable was the reconstructed binomial data corresponding to the estimated multilocus outcrossing rate and sample size per population (see Materials and methods). The ecological covariates were openness (closed versus open), water regime (permanent versus temporary), the harmonic mean density $\left(d_{\text {harm }}\right)$, the frequency of occurrence of densities $\leq 1$ ind $\mathrm{m}^{-2}\left(d_{\text {freq }}\right)$ and the density at sampling $\left(d_{\text {sampl }}\right)$. All effects were tested against the null model.

more frequent in open temporary $(45 \%, N=42$ visits) than in closed permanent $(36 \%, N=72)$, closed temporary $(10 \%, N=62)$ and open permanent sites $(8 \%, N=40)$.

None of the predictions of the RAH (Table 2) were supported by our results. Although variation in outcrossing probability could be explained by habitat characteristics (interaction Openness*Water regime, Table 4), they did not fit with variation in $d_{\text {freq, }}$ at variance with predictions from the RAH: the overall outcrossing rate was lower in closed permanent (0.86) than in open temporary (0.92), closed temporary (0.95) and open permanent (0.98). Moreover, this variation was no more significant when tested in the presence of $d_{\text {sampl }}$ (results not shown; F1 and F2 not fulfilled). $d_{\text {harm }}$ and $d_{\text {freq }}$ did not explain the among-population variation in outcrossing probability (Table 4; F3 and F4 not fulfilled).

The population-level probability of outcrossing was negatively dependent on the density at sampling (Table 4; Figure 1), contrary to the predicted positive correlation (P1). This effect remained strongly significant when using a more conservative statistical approach under which the sample size per population was reconstructed such as to be proportional to the SD of $t_{\mathrm{mp}}$ (results not shown). At the population level, multiple paternities occurred in the three populations in which significant selfing was detected (Table 1). At the family level, there was no relationship between $t_{\mathrm{mf}}$ and $r_{\mathrm{p}} \quad(\mathrm{r}=0.088$, d.f. $=98, \quad P=0.385$; P2 not fulfilled). The egg-laying delay did not depend on the family-level multilocus outcrossing rate (Table 3; prediction P3), although essentially selfing individuals tended to exhibit long egg-laying delays.

\section{Discussion}

The mating system of $P$. acuta

$P$. acuta reproduced primarily through outcrossing, with a population-level average outcrossing rate of 0.936 . Over all families, the average outcrossing rate was 0.885 . These results are in agreement with those in a natural population from the same area (0.921 in Jarne et al, 2000). Wethington and Dillon (1997) found a rather lower value (0.385), but this was under laboratory conditions with North-American individuals. Our estimates based on $F_{\text {is }}$

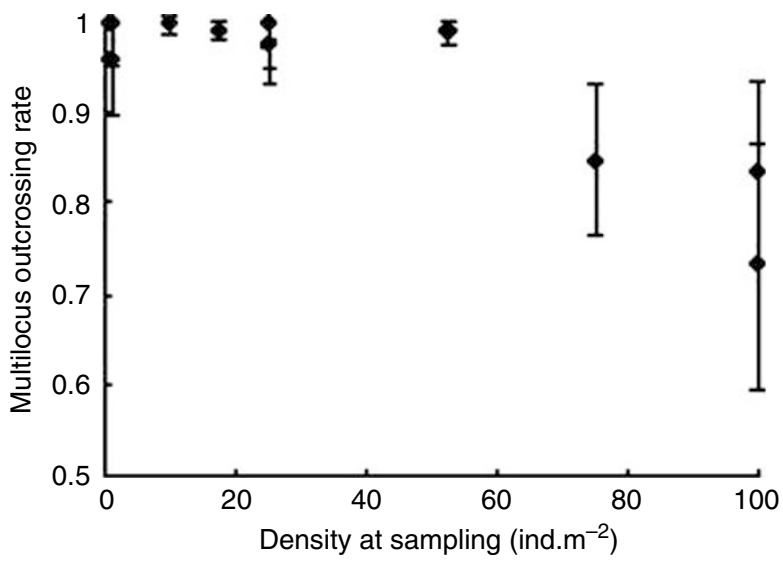

Figure 1 Relationship between population-level multilocus outcrossing rate $\left(t_{\mathrm{m}} \pm \mathrm{SD}\right)$ and population density at sampling $\left(d_{\text {sampl }}\right)$ in $P$. acuta $(N=11$ populations).

(average $t_{F_{\mathrm{is}}}: 0.89$ ) were close to those derived from progeny arrays.

Examining fitness components showed that the variation in the proportion of fertile individuals, number of eggs per capsule, incubation time, and offspring survival were not explained by variation in outcrossing rate. This might be interpreted as evidence of limited inbreeding depression. However, this would be surprising since strong inbreeding depression has been consistently detected in P. acuta (Wethington and Dillon, 1997; Jarne et al, 2000; Henry et al, 2003). Moreover, in the partially selfing population Lam12, $F_{\text {is }}$ did not differ from zero, suggesting the occurrence of inbreeding depression between the juvenile and adult stages. Alternatively, this might be due to imprecision in family-level estimates of $t_{\mathrm{mf}}$ which is not accounted for in our analyses. Discounting the estimation error on $t_{\mathrm{mf}}$ blurs the acceptance of the null hypothesis. To circumvent this general problem, a comprehensive statistical framework is required that would allow comparative analyses of mating system parameters, and in which the contribution of individual and population covariates could be directly built in the Maximum Likelihood procedure and tested through comparisons of nested models.

\section{Tests of the RAH}

The RAH has been repeatedly invoked to explain the maintenance of selfing in sessile or poorly mobile organisms. Its role was suggested in freshwater snails because these organisms often inhabit unpredictable temporary habitats (eg Jarne and Charlesworth, 1993; Städler and Jarne, 1997; Charbonnel et al, 2002). Tsitrone et al (2003a) clarified the conditions under which RA is likely to play a role in mainly outcrossing animal species (see Introduction). Their model has been supported by experimental work conducted in P. acuta that showed that the waiting time is heritable (Tsitrone et al, 2003b). There is therefore evidence from a direct approach that the RAH explains residual selfing in highly outcrossing species.

The RAH was here evaluated using an indirect, correlative approach. None of the tests performed (Table 2) support the hypothesis that P. acuta self- 
fertilizes in response to a low probability of mate encounter. At the population level, the ecological constraints on the mating system related to habitat, via population density, were not those expected: temporary or closed habitats were no more likely to go regularly through low-density periods than permanent or open habitats (the opposite trend was indeed found). A conservative interpretation of our results is that the outcrossing rate was not related to those environmental variables that might influence the probability of mate encounter, that is, water regime, habitat openness, temporal fluctuations of density, and occurrence of low-density events. The density of $\mathrm{G}_{0}$ individuals at sampling did explain a significant fraction of the amongpopulation variation in outcrossing rate. However, the relationship was opposite to the prediction: increasing population density was associated with decreasing outcrossing rate. Another surprising result under the RAH is that multiple paternity and the selfing rate were positively associated: why did individuals self their own ovules when mate density was high enough to ensure multiple paternities in some offspring (see also Cheptou et al, 2002)? On the whole, the indirect approach developed here did not provide any evidence in favour of the RAH as a major determinant of the selfing rate. This is compatible with results from another indirect approach in which the probability of mate encounter was experimentally manipulated (Henry, 2002).

It must be emphasized that our predictions on the probability of mate encounter were based on short-term ecological trends and population dynamics (over a 2-year survey). This did not capture the occurrence of much rarer events of low mate encounter probability, that is, due to abnormal disturbance events (eg, prolonged drying out) or very rare founding events. Those events might have indeed been the ecological factors maintaining selfing as a RA mechanism. Founding events very likely occur in $P$. acuta since this species displays high dispersal abilities (reviewed in Henry, 2002) and is the most successful worldwide invasive freshwater snail (Dillon et al, 2002; Bousset and Jarne, unpublished data). Such founding events might be scarce in the studied area, since neither demographic, nor genetic signatures were detected (Henry, 2002; Bousset et al, 2004). The mating system would rapidly revert to preferential outcrossing when mates become available, because of inbreeding depression (Stephenson et al, 2000; Pannell and Barrett, 2001), and signals of RA would no more be detectable.

Very few studies in animals have evaluated the $\mathrm{RAH}$, and most failed. In predominantly selfing snails, Trouvé et al (2003) and Charbonnel et al (2005) concluded that the among-population variation in selfing rate is inconsistent with this hypothesis. The only supporting results come from comparative analyses showing that selfing gastropods are better colonizers (Selander and Ochman, 1983; Bengtsson and Baur, 1993). Less controversial arguments come from studies in plants, where negative relationships between self-pollination (and morphological or incompatibility-related traits) and density (of plants or of pollination vectors) have been repeatedly documented (eg Rankin et al, 2002 and references therein; but see eg Cheptou et al, 2002 for opposite results). However, it is worth noting that most results are derived from correlative observations of fitness components (the indirect approach above). Moreover, the only satisfactory quantitative test dismissed the evolutionary importance of RA because the advantage of autogamy was outweighed by seed discounting and inbreeding depression (Herlihy and Eckert, 2002). On the whole, the significance of selfing as a RA strategy, particularly in animals, remains to be demonstrated.

\section{So, why do $P$. acuta and other snail species partially self-fertilize?}

Although several studies have dealt with the reproductive biology of P. acuta, the maintenance of some selfing remains unexplained (Henry, 2002; but see Tsitrone et al, $2003 b)$. We briefly review several theories, which are alternatives to the RAH, which may be consistent with the empirical evidence from $P$. acuta. These explanations can be extended to other freshwater snail species with a mating system similar to that of P. acuta (see Henry et al, 2003 for a list). A first view assumes that partial selfing in P. acuta is the expression of a mixed-mating system, that is, a polymorphism with genetically fixed selfing rates. Three evolutionary scenarios can be envisaged. (i) The selfing rate greatly varies among families (Jarne et al, 2000; this study; see Trouvé et al, 2003 and references therein for results in other snail species), as do selfing propensity and inbreeding depression (Jarne et al, 2000; Henry et al, 2003; Tsitrone et al, 2003b). The required conditions for a coevolution between loci determining the selfing rate and fitness driving to mixed mating therefore seem to be met (Uyenoyama et al, 1993). However, the observation that no genetic covariation between selfing propensity and inbreeding depression was found in P. acuta (Escobar, David and Jarne, unpublished data) weakens this hypothesis. (ii) Biparental inbreeding, due to restricted dispersal in association with density-dependent recruitment (Uyenoyama, 1986; Ronfort and Couvet, 1995), could also be relevant and was detected in five populations out of 11 in this study. However, significant selfing rates were not systematically associated with biparental inbreeding, and density-dependent recruitment, although likely, remains to be investigated in $P$. acuta. (iii) Mixed mating can evolve in structured populations (eg Pannell and Barrett, 2001). The highly fragmented, potentially unstable populations of P. acuta, in association with preferential outcrossing, high dispersal abilities and high inbreeding depression, nicely fit the situation modelled by Pannell and Barrett (2001): in the case of extinctioncolonization, preferential outcrossing should have a selective advantage over preferential selfing thanks to a higher number of viable propagules.

A second view envisages the selfing rate as a plastic trait, and plasticity itself as the target of natural selection, adjusted to environmental conditions (Stephenson et al, 2000; Pannell and Barrett, 2001). Tsitrone et al (2003a) demonstrated that delaying selfing according to mate availability increases both the probability of mate encounter and future reproduction through resource reallocation towards growth. A side prediction is that, if the waiting time before selfing is positively related to survival, the propensity to self-fertilize should be higher when survival is low. If high population density represents a high mortality risk, this would explain why the outcrossing rate was here negatively related to 
population density. Also, it could explain within-individual temporal variation, like age dependency, in selfing rate (Watkins and Levin, 1990; Stephenson et al, 2000; Rankin et al, 2002), which remains to be documented in hermaphroditic animals.

\section{Acknowledgements}

We are very grateful to $\mathrm{N}$ Charbonnel, $\mathrm{P}-\mathrm{O}$ Cheptou, $\mathrm{R}$ Williams and two anonymous reviewers for comments on the manuscript, to $\mathrm{T}$ Lenormand for statistical advices, and to the Service Commun des Marqueurs Génétiques at CEFE, especially C Debain, for technical support with the genetic analyses. This project was funded by grants to PJ from the Centre National de la Recherche Scientifique (Programme Jeune Equipe), the Ministère de $1^{\prime}$ Ecologie et du Développement Durable (Programme InvaBio) and the Bureau des Ressources Génétiques. PYH benefitted from a fellowship from the Ministère de l'Education Nationale, de la Recherche et de la Technologie, and LB from an ASC fellowship from the Institut National de la Recherche Agronomique.

\section{References}

Barrett SCH (2002). The evolution of plant sexual diversity. Nat Rev Genet 3: 274-284.

Bengtsson J, Baur B (1993). Do pioneers have r-selected traits? Life history patterns among colonizing terrestrial gastropods. Oecologia 94: 17-22.

Bousset L, Henry PY, Sourrouille P, Jarne P (2004). Population biology of the invasive freshwater snail Physa acuta approached through genetic markers, ecological characterization and demography. Mol Ecol 13: 2023-2036.

Charbonnel N, Lefebvre O, Sellin E, Jarne P (2005). The influence of genetic factors and population dynamics on the mating system of the hermaphroditic freshwater snail Biomphalaria pfeifferi. Oikos 108: 283-296.

Charbonnel N, Quesnoit M, Razatavonjizay R, Bremond P, Jarne $P$ (2002). A spatial and temporal approach to microevolutionary forces affecting population biology in the freshwater snail Biomphalaria pfeifferi. Am Nat 160: 741-755.

Cheptou P-O, Dieckmann U (2002). The evolution of selffertilization in density-regulated populations. Proc R Soc Lond B Biol Sci 269: 1177-1186.

Cheptou P-O, Lepart J, Escarré J (2002). Mating system variation along a successional gradient in the allogamous and colonizing plant Crepis sancta (Asteraceae). J Evol Biol 15: 753-762.

Dillon RTJ, Wethington AR, Rhett JM, Smith TP (2002). Populations of the European freshwater pulmonate Physa acuta are not reproductively isolated from American Physa heterostropha or Physa integra. Invertebr Biol 121: 226-234.

Dole JA (1992). Reproductive assurance mechanisms in three taxa of the Mimulus guttatus complex (Scrophulariaceae). Am J Bot 79: 650-659.

Fausto JAJ, Eckhart VM, Geber MA (2001). Reproductive assurance and the evolutionary ecology of self-fertilization in Clarkia xantiana (Onagraceae). Am J Bot 88: 1794-1800.

Henry PY (2002). Dynamique des populations et évolution $d u$ système de reproduction. Etude chez le gastéropode hermaphrodite Physa acuta (Pulmoné). PhD thesis, Université Montpellier II, France.

Henry PY, Pradel R, Jarne P (2003). Environment-dependent inbreeding depression in a hermaphroditic freshwater snail. J Evol Biol 16: 1211-1222.

Herlihy CR, Eckert CG (2002). Genetic cost of reproductive assurance in a self-fertilizing plant. Nature 416: 320-323.
Jarne P, Charlesworth D (1993). The evolution of the selfing rate in functionally hermaphrodite plants and animals. Annu Rev Ecol Syst 24: 441-466.

Jarne P, Perdieu M-A, Pernot A-F, Delay B, David P (2000). The influence of self-fertilization and grouping on fitness attributes in the freshwater snail Physa acuta: population and individual inbreeding depression. J Evol Biol 13: 645-655.

Lande R, Schemske DW (1985). The evolution of self-fertilization and inbreeding depression in plants. I. Genetic models. Evolution 39: 24-40.

Lloyd DG (1980). Demographic factors and mating patterns in Angiosperms. In: Solbrig OT (ed) Demography and Evolution in Plant Populations. Blackwell: Oxford. pp 67-88.

Lloyd DG (1992). Self- and cross-fertilization in plants. II. The selection of self-fertilization. Int J Plant Sci 153: 370-380.

Meunier C, Hurtrez-Bousses S, Jabbour-Zahab R, Durand P, Rondelaud D, Renaud F (2004). Field and experimental evidence of preferential selfing in the freshwater mollusc Lymnaea truncatula (Gastropoda, Pulmonata). Heredity 92 316-322.

Monsutti A, Perrin N (1999). Dinucleotide microsatellite loci reveal a high selfing rate in the freshwater snail Physa acuta. Mol Ecol 8: 1076-1078.

Mood AM, Graybill FA, Boes DC (1974). Introduction to the Theory of Statistics. Mac-Graw-Hill: New York.

Pannell JR, Barrett SCH (1998). Baker's law revisited: reproductive assurance in a metapopulation. Evolution 52: 657-668.

Pannell JR, Barrett SCH (2001). Effects of population size and metapopulation dynamics on a mating-system polymorphism. Theor Popul Biol 59: 145-155.

Pollak E (1987). On the theory of partially inbreeding finite populations. I. Partial selfing. Genetics 117: 353-360.

Rankin AE, Weller SG, Sakai AK (2002). Mating system instability in Schiedea menziesii (Caryophyllaceae). Evolution 56: $1574-1585$.

Ritland K (2002). Extension of models for the estimation of mating systems using $n$ independent loci. Heredity 88: 221-228.

Ronfort J, Couvet D (1995). A stochastic model of selection on selfing rates in structured populations. Genet Res 65: 209-222.

Schoen DJ, Morgan MT, Bataillon T (1996). How does selfpollination evolve? Inferences from floral ecology and molecular genetic variation. Philos Trans $R$ Soc Lond B Biol Sci 351: 1281-1290.

Selander RK, Ochman H (1983). The genetic structure of populations as illustrated by molluscs. Isozymes 10: 93-123.

Sourrouille P, Debain C, Jarne P (2003). Microsatellite variation in the freshwater snail Physa acuta. Mol Ecol Notes 3: 21-23.

Städler T, Jarne P (1997). Population biology, genetic structure, and mating system parameters in freshwater snails. In: Streit B, Städler T, Lively CM (eds) Ecology and Evolution of Freshwater Organisms. Birkhäuser: Basel. pp 231-262.

Stephenson AG, Good SV, Vogler DW (2000). Interrelationships among inbreeding depression, plasticity in the self-incompatibility system, and the breeding system of Campanula rapunculoides L. (Campanulaceae). Ann Bot London 85: S211-S219.

Sun M, Ritland K (1998). Mating system of yellow starthistle (Centaurea solstitialis), a successful colonizer in North America. Heredity 80: 225-232.

Trouvé S, Degen L, Renaud F, Goudet J (2003). Evolutionary implications of a high selfing rate in the freshwater snail Lymnaea truncatula. Evolution 57: 2303-2314.

Tsitrone A, Duperron S, David P (2003a). Delayed selfing as an optimal mating strategy in preferentially outcrossing species: theoretical analysis of the optimal age at first reproduction in relation to mate availability. Am Nat 162: 318-331.

Tsitrone A, Jarne P, David P (2003b). Delayed selfing and resource reallocations in relation to mate availability in the freshwater snail Physa acuta. Am Nat 162: 474-488. 
Uyenoyama MK (1986). Inbreeding and the cost of meiosis: the evolution of selfing in populations practicing biparental inbreeding. Evolution 40: 388-404.

Uyenoyama MK, Holsinger KE, Waller DM (1993). Ecological and genetic factors directing the evolution of self-fertilization. Oxford Surv Evol Biol 9: 327-381.
Watkins L, Levin DA (1990). Outcrossing rates as related to plant density in Phlox drummondii. Heredity 65: 81-89.

Wethington AR, Dillon RTJ (1997). Selfing, outcrossing, and mixed mating in the freshwater snail Physa heterostropha: lifetime fitness and inbreeding depression. Invertebr Biol 116: 192-199.

Supplementary Information accompanies the paper on Heredity website (http://www.nature.com/hdy) 